\title{
The Burden of Hidradenitis Suppurativa in a Cohort of Patients in Southern Finland: A Pilot Study
}

\author{
Nicolas Kluger a, b Martta Ranta ${ }^{c}$ Martina Serlachius ${ }^{c}$ \\ a University of Helsinki, and ${ }^{\mathrm{b}}$ Department of Dermatology and Allergology, Helsinki University Central Hospital, \\ Helsinki, and ${ }^{\mathrm{C}} \mathrm{AbbVie}$ Oy, Espoo, Finland
}

\section{Keywords}

Hidradenitis suppurativa - Disease burden - Quality of life · Finland

\begin{abstract}
Background: Hidradenitis suppurativa (HS) is a chronic inflammatory disease that impacts the quality of life. Methods: We evaluated its burden in a cohort of 26 Finnish patients in a single-center cross-sectional study. Demographic data, disease history, clinical data, treatment patterns, and workability were collected. Patients responded to the Dermatology Life Quality Index (DLQI) and Beck Depression Inventory (BDI-21). Results: Mean age of HS patients was 44.2 years and mean diagnostic delay was 13.7 years. Pain was rated the most bothersome symptom. Mean DLQI score was 8.31. Women consistently had higher scores than men for every item of the DLQI. Mean BDI-21 score was 10.69. Higher Hurley stage at visit $(p=0.001)$, female gender $(p=0.018)$, and higher BDI-21 score ( $p=0.022)$ were variables that significantly affected the total DLQI score, whereas female gender ( $p=0.004$ ) was the only variable that significantly affected the total BDI-21 score using stepwise regression analysis. Conclusions: The burden of HS is for the first time reported in Finland. The diagnostic delay is longer than previously reported and the results suggest that women are impacted by the disease more than men.

๑) 2017 S. Karger AG, Basel
\end{abstract}

\section{Introduction}

Hidradenitis suppurativa (HS) is a chronic inflammatory disease of the terminal hair follicles, affecting the intertriginous skin area of axillary, genitofemoral, and perianal sites [1]. The heterogeneous clinical manifestations of HS may lead to misdiagnosis and delay in disease management. The precise prevalence of HS remains unknown because of the difficulty in collecting and extrapolating data [2]. In Denmark, the prevalence of HS varies between 1 and 4\%, depending on the study setting [2]. The numerous comorbidities that are associated with HS contribute to the impaired quality of life (QoL) in patients with HS [3]. To date, no published data exists on the clinical profile and burden of disease in Finnish patients with HS. Thus, we performed a cross-sectional pilot study to assess the burden of HS in a cohort of 26 Finnish patients.

\section{Methods}

Study Population

The Ethical Committee of HUCH Internal Medicine approved the study (191/13/03/01/15) and each participant provided informed consent.

A single-center cross-sectional study was conducted on 26 adult patients with a confirmed HS diagnosis (Fig. 1). Briefly, 69 patients with a diagnosis of HS (ICD-10 diagnosis code L73.2), seen by physicians at the Department of Dermatology in the Hospital District

\section{KARGER}

(c) 2017 S. Karger AG, Basel 


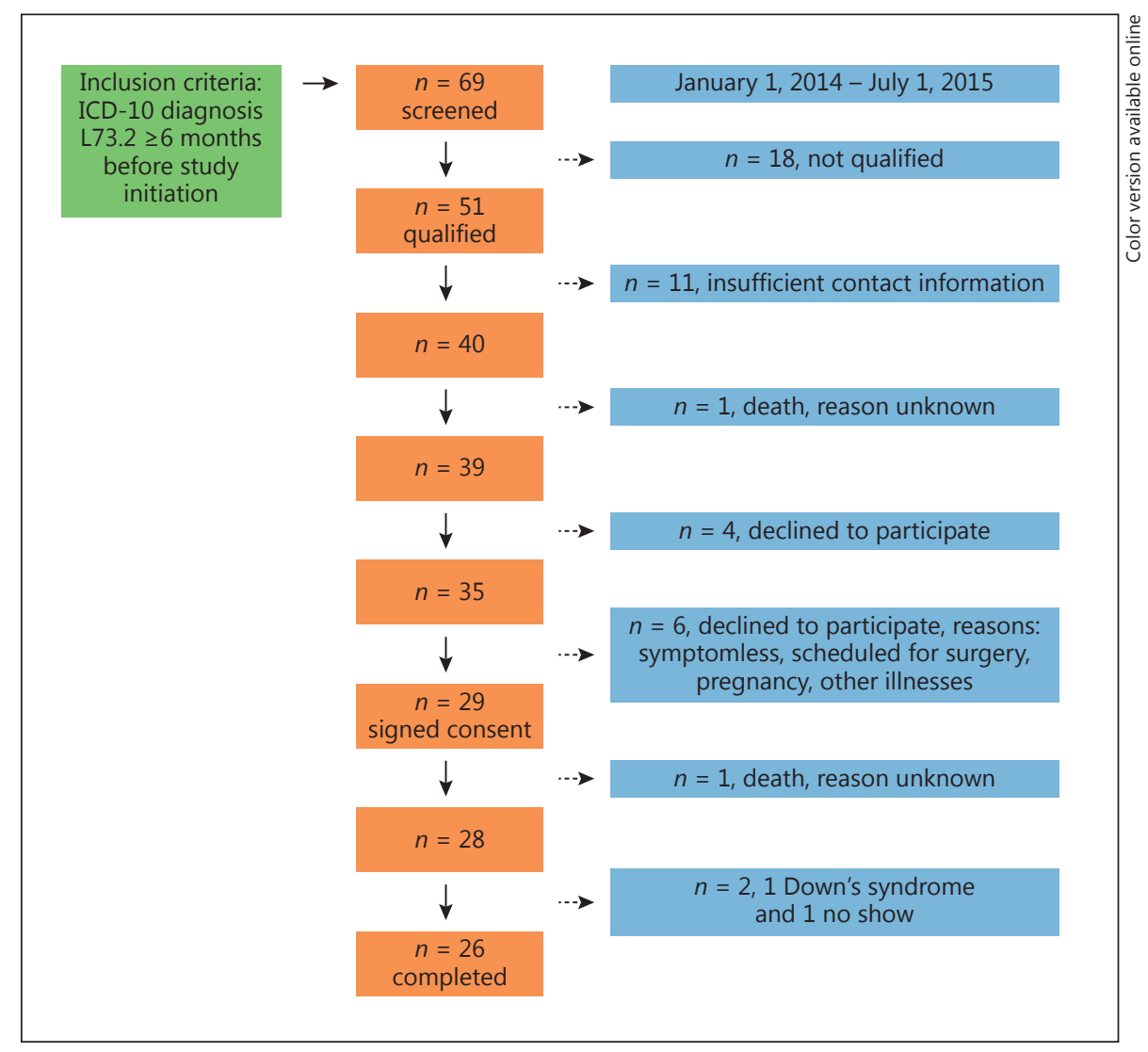

Fig. 1. Flowchart of patient selection.

of Helsinki and Uusimaa between January 1, 2014, and July 1, 2015, were identified. The principal investigator (N.K.) reviewed all medical files for inclusion into the study: patients $\geq 18$ years of age with HS diagnosed $\geq 6$ months before study initiation with the ability to provide informed consent. All three diagnostic criteria for HS had to be met: presence of typical lesions, location of lesions in typical areas, and an evolving disease course with relapses and chronicity. Of 69 identified patients, 18 did not meet the eligibility criteria. Of 51 eligible patients, 11 could not be contacted due to insufficient contact information and 1 died. Of the remaining 39 patients, 4 declined to participate and 6 did not participate for the following reasons: currently symptomless, scheduled for surgery, pregnancy, and other illness. Altogether, 29 patients provided consent to participate in the study. Of these, 1 died before the study visit, 1 had Down's syndrome and was not able to answer the questionnaires herself, and 1 did not show up for the study visit. In total, 26 patients were included in the study. The study was divided into 3 sections: chart review, patient questionnaires, and study visit to the physician. To protect patient confidentiality, all personally identifiable information was de-identified in all 3 sections.

Chart Review and Study Visit to the Physician

Medical charts of eligible patients were screened. Data were collected on demographics, social and economic status, personal and familial history of HS, lifestyle, comorbidities, clinical presentation and severity of HS, and burden on the healthcare system (e.g., HS-related surgeries).

Hidradenitis Suppurativa in a Finnish Cohort
During the study visit, the principal investigator (N.K.) assessed current disease status (i.e., disease severity according to Hurley stage and clinical presentation) and collected information on treatment patterns and HS-related sick time over the past year. Patients were also asked to rank 10 HS-related symptoms according to the impact on their everyday life. The most bothersome symptom was scored 1 and the least bothersome symptom was scored 10 .

\section{Questionnaires}

Patients were asked to complete a comprehensive set of questionnaires (Work Productivity and Activity Impairment-Specific Health Problem [WPAI-SHP], Dermatology Life Quality Index [DLQI], Beck Depression Inventory [BDI-21], 15D Instrument [15D], Hidradenitis Suppurativa Symptom Assessment [HSSA], and Hidradenitis Suppurativa Impact Assessment [HSIA]) at home before the study visit. The results of the DLQI, BDI-21, and WPAI-SHP are presented here.

The DLQI is the most frequently used instrument in randomized controlled trials in dermatology [4]. It is a simple, validated questionnaire that consists of 10 questions. Each question has 4 possible answers for a maximum of 3 points and a total maximum score of 30 . Higher score indicates more severely affected QoL. A total score of $0-1$ indicates no effect on QoL, 2-5 indicates a small effect, 6-10 indicates a moderate effect, 11-20 indicates a very large effect, and 21-30 indicates an extremely large effect $[4,5]$.

The BDI-21 is one of the most widely used instruments for measuring depression [6]. We consulted a psychiatrist, who veri- 
Table 1. Demographics, comorbidities, and clinical characteristics in a cohort of Finnish patients suffering from hidradenitis suppurativa

\begin{tabular}{|c|c|c|c|}
\hline & Total & Men & Women \\
\hline \multicolumn{4}{|l|}{ Demographics } \\
\hline Number of patients & 26 & $10(38.5 \%)$ & $16(61.5 \%)$ \\
\hline Age, years & $44.2 \pm 15.5$ & $49.4 \pm 12.9$ & $40.9 \pm 14.9$ \\
\hline Age at onset, years & $26.8 \pm 14.9$ & $27.1 \pm 14.1$ & $26.6 \pm 16.0$ \\
\hline Age at diagnosis, years & $40.5 \pm 15.0$ & $44.4 \pm 14.5$ & $38.2 \pm 15.3$ \\
\hline Duration of hidradenitis suppurativa, years & $18.4 \pm 10.2$ & $21.1 \pm 13.1$ & $16.7 \pm 8.0$ \\
\hline Body mass index & $31.2 \pm 7.31$ & $31.9 \pm 6.8$ & $30.7 \pm 7.5$ \\
\hline \multicolumn{4}{|l|}{ Smoking } \\
\hline Smoker & $20 / 26(76.9 \%)$ & $10 / 10(100 \%)$ & $10 / 16(62.5 \%)$ \\
\hline Present smoker & $10 / 26(38.5 \%)$ & $5 / 10(50 \%)$ & $5 / 16(31.25 \%)$ \\
\hline Past smoker & $10 / 26(38.5 \%)$ & $5 / 10(50 \%)$ & $5 / 16(31.25 \%)$ \\
\hline Never smoker & $6 / 26(23.1 \%)$ & $0 / 10(0 \%)$ & $6 / 16(37.5 \%)$ \\
\hline Pack-years & $12.9 \pm 13.0$ & $21.0 \pm 12.6$ & $7.8 \pm 10.7$ \\
\hline \multicolumn{4}{|l|}{ Alcohol consumption } \\
\hline Alcohol consumers & $19 / 26(73.1 \%)$ & $9 / 10(90 \%)$ & $10 / 16(62.5 \%)$ \\
\hline Quantity, g/week & $31.2 \pm 63.5$ & $63.0 \pm 94.3$ & $11.4 \pm 16$ \\
\hline \multicolumn{4}{|l|}{ Comorbidities } \\
\hline Obesity (body mass index $>30$ ) & $13 / 26(50 \%)$ & $5 / 10(50 \%)$ & $8 / 16(50 \%)$ \\
\hline Type II diabetes & $4 / 26(15.4 \%)$ & $3 / 10(30 \%)$ & $1 / 16(6.2 \%)$ \\
\hline Cardiovascular diseases & $9 / 26(34.6 \%)$ & $6 / 10(60 \%)$ & $3 / 16(18.7 \%)$ \\
\hline Autoimmune or inflammatory diseases ${ }^{\mathrm{a}}$ & $5 / 26(19.2 \%)$ & $3 / 10(30 \%)$ & $2 / 16(12.5 \%)$ \\
\hline Depression/anxiety disorders & $4 / 26(15.4 \%)$ & $0 / 10(0 \%)$ & $4 / 16(25 \%)$ \\
\hline \multicolumn{4}{|l|}{ Clinical characteristics } \\
\hline \multicolumn{4}{|l|}{ Hurley stage } \\
\hline Stage I & $13 / 26(50 \%)$ & $2 / 10(20 \%)$ & $11 / 16(68.8 \%)$ \\
\hline Stage II & $11 / 26(42.3 \%)$ & $7 / 10(70 \%)$ & $4 / 16(25 \%)$ \\
\hline Stage III & $2 / 26(7.7 \%)$ & $1 / 10(10 \%)$ & $1 / 16(6.2 \%)$ \\
\hline Mean number of affected areas & $2.04 \pm 1.58$ & $2.10 \pm 1.90$ & $2.00 \pm 1.38$ \\
\hline \multicolumn{4}{|l|}{ Number of affected areas } \\
\hline 1 & $10 / 26(38.5 \%)$ & $2 / 10(20 \%)$ & $8 / 16(50 \%)$ \\
\hline 2 & $7 / 26(26.9 \%)$ & $5 / 10(50 \%)$ & $2 / 16(12.5 \%)$ \\
\hline 3 & $7 / 26(26.9 \%)$ & $3 / 10(30 \%)$ & $4 / 16(25 \%)$ \\
\hline 4 & $2 / 26(7.7 \%)$ & $0 / 10(0 \%)$ & $2 / 16(12.5 \%)$ \\
\hline
\end{tabular}

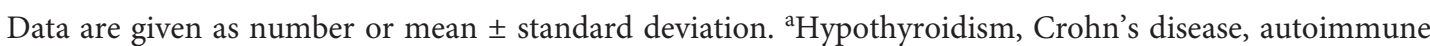
cholangitis, myasthenia, and cutaneous lupus.

fied that the BDI-21 questionnaire is suitable for this type of study. The questionnaire is composed of items relating to depressive, cognitive, and physical symptoms. The BDI-21 consists of 21 questions and each question has a set of at least 4 possible answers, ranging in intensity. A value of $0-3$ is assigned for each answer. Higher total scores indicate more severe depressive symptoms according to the following grade [7]: normal (0-12 points), mild depression (13-18 points), moderate depression (19-29 points), and severe depression ( $\geq 30$ points).

\section{Statistical Analysis}

Statistical analysis was conducted using SPSS Statistics 22 (IBM, Armonk, NY, USA) and SAS ${ }^{\circledR}$ version 9.3 for Windows (SAS Institute, Cary, NC, USA). Patient characteristics are presented as means and standard deviations for continuous variables and as frequencies and proportions for categorical variables. Patient characteristics were compared using the $\chi^{2}$ test for categorical variables and the MannWhitney $U$ test for continuous variables. Correlations between age, age at diagnosis, duration of the disease, level of education, number of HS-affected locations, Hurley staging, comorbidities, and total DLQI and BDI-21 scores were analyzed using the Spearman rank correlation coefficient. A stepwise regression analysis estimated how gender, smoking (pack-years), alcohol consumption (grams per week), body mass index, overall number of comorbidities, and severity of the disease (Hurley staging, number of affected locations) affected the variance in the BDI-21 and DLQI scores. The statistical regression threshold was set at $p=0.15$ for inclusion into the model. $p<0.05$ (2-sided) was considered statistically significant. 
Fig. 2. Self-reported bothersome symptoms of HS. Patients were given a list of 10 HS-related symptoms. They were asked to rank them according to the impact on their everyday life. A score of 1 was given to the most bothersome symptom and a score of 10 was given to the least bothersome symptom. Results are presented as means of the scores given to each symptom by each patient and ranked from 1 to 10 accordingly.

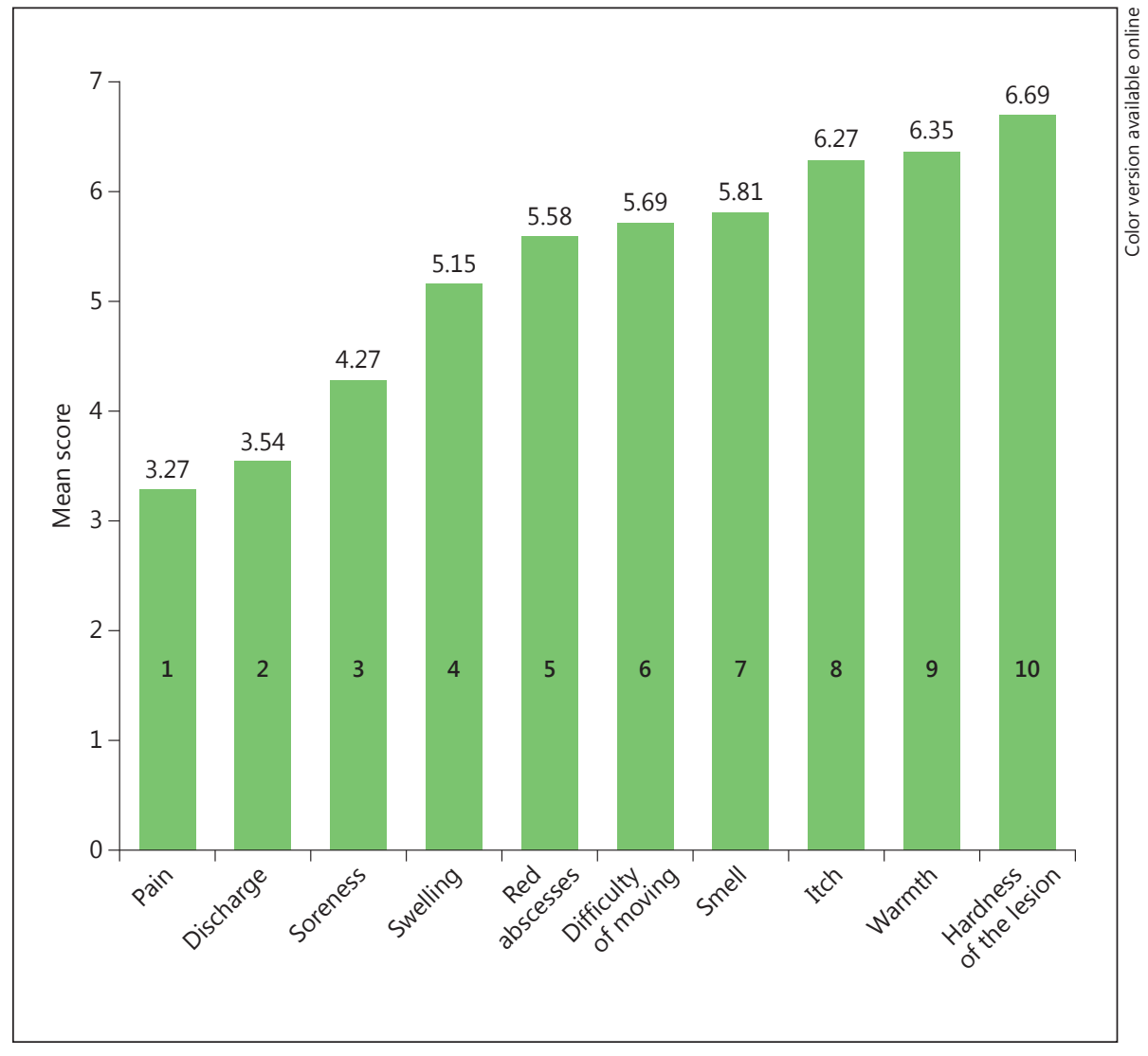

\section{Results}

\section{Demographic Data}

Of 26 patients with HS, 10 were men (38.5\%) and 16 were women (61.5\%); mean age of patients was $44.2 \pm 15.5$ years (range, 23-72 years). Totally, 96.2\% (25/26) were white; 1 was of African descent. Men were slightly older (49.4 \pm 12.9 years; range, $29-66$ years) than women (40.9 \pm 14.9 years; range, $23-72$ years). Mean age at HS diagnosis was $40.5 \pm 15.0$ years (range, $18-70$ years); however, the mean age at onset of HS was $26.8 \pm 14.9$ years (range, 8-66 years). Mean average disease duration was $18.4 \pm 10.2$ years (Table 1). There were no statistically significant differences between genders for age, age at onset, age at diagnosis, or disease duration. Family history of HS was reported in less than a third of the cases $(30.4 \%$; $7 / 23)$, of which 6 cases $(85.7 \%)$ were in first-degree relatives (parents or siblings, including 1 twin sister). In one case, a second-degree relative was affected (aunt). More than half (57.5\%) had a high school or higher educational degree and $58.3 \%$ had an annual income between EUR 25,000 and 59,999. Totally, 16 patients (61.5\%) were employed.

\section{Clinical Presentation of HS}

Medical file information on clinical presentation and disease severity was limited. At the time of interview, 13 patients $(50.0 \%)$ had Hurley stage I, 11 (42.3\%) had Hurley stage II, and 2 (7.7\%) had Hurley stage III (Table 1). The body regions most commonly affected were the inguinal (76.9\%) and axillary (61.5\%) regions, as well as the buttocks (11.5\%). Mean number of affected areas was $2.04 \pm 1.58$ (range, $1-4$ ). In men, the inner thigh/groin area was affected in $80 \%$ of the cases, followed by the armpits (60\%) and buttocks (40\%). Among women, the inner thigh/groin area was affected in $75.0 \%$ of cases, followed by the armpits $(62.5 \%)$, buttocks $(25.0 \%)$, and breasts (18.8\%). No significant differences were observed between men and women ( $\chi^{2}$ test).

\section{Lifestyle and Comorbidities}

All men were present or past smokers, while 37.5\% $(6 / 16)$ of women had never smoked. Men smoked more than women (Mann-Whitney U test, $p=0.006$ ). Alcohol consumption was more frequent among men $(90.0 \%$ of men $[9 / 10]$ vs. $62.5 \%$ of women [10/16]), men also con- 
sumed more alcohol weekly (Mann-Whitney U test, $p=$ 0.02) (Table 1).

Mean number of comorbidities was $1.35 \pm 1.3$ (1.7 \pm 1.4 for men and $1.1 \pm 1.1$ for women). Half of the patients $(50.0 \% ; 13 / 26)$ were obese (body mass index $[\mathrm{BMI}]>30$; mean BMI, $31.2 \pm 7.3$; range, $17.9-47)$ and 3 patients were morbidly obese (BMI $>40)$. Only $15.4 \%$ of patients $(4 / 26)$ had a BMI $<25$ (i.e., normal weight) and 1 patient was underweight (BMI <18). Sixty percent of men (vs. 18.7\% of women) disclosed a cardiovascular morbidity ( $\chi^{2}$ test, $p=0.031)$. There was no gender difference regarding the prevalence of other comorbidities (Table 1). A past history of acne, sinus pilonidalis, and polycystic ovary syndrome was noted in $30.8 \%(8 / 26), 15.4 \%(4 / 26)$, and $6.25 \%$ ( $1 / 16$ women) of patients, respectively.

\section{Pain, Quality of Life, and Depression}

Patients were asked to rank a list of 10 HS-related symptoms according to the impact on their everyday life. Results are presented as means of the symptom scores as rated by each patient (Fig. 2). Pain was ranked as the most bothersome HS-related symptom (mean score, 3.27).

Mean DLQI score was $8.31 \pm 7.39$ (3.00 \pm 3.77 for men and $11.63 \pm 7.22$ for women). The difference was statistically significant between men and women, with women having higher scores (Mann-Whitney $\mathrm{U}$ test, $p=0.002$ ). Nine patients (34.6\%), all women, reported a very large to extremely large effect on their daily life (DLQI $>10)$. The items most affected were skin symptoms and embarrassment (38.5\%), problems with clothing (36.0\%), and impaired sexuality (26.6\%; Fig. 3a). Women had higher scores than men for every DLQI item. The most notable difference between men and women was observed for the embarrassment item $(p<0.001)$. In addition, scores for clothing $(p=0.010)$, problems with partners, relatives, or close friends $(p=0.004)$, and sexuality $(p=0.007)$ were statistically significantly higher in women than men (Fig. 3b). Skin symptoms $(p=0.052)$ and social/leisure activities $(p=0.070)$ were affected, but without significant inter-gender differences. Higher Hurley stage at visit $(p=$ $0.001)$, female gender $(p=0.018)$, and higher BDI-21 total score $(p=0.022)$ were variables that significantly affected the total DLQI score in stepwise regression analysis.

Mean total BDI-21 score was $10.69 \pm 10.13$. Women had higher scores than men (mean, $15.00 \pm 10.32$ vs. $3.80 \pm$ 4.66; Mann-Whitney U test, $p=0.002)$. According to BDI21 severity grading, 10 patients $(38.5 \%)$, of which 9 were women, reported depression symptoms (BDI-21 >12); mild, moderate, and severe depression were reported in 6 , 2 , and 2 patients, respectively. Four patients reported de- pression during the interview. BDI-21 scores did not correlate with the age of the patient, the age at diagnosis, HS duration, the number of affected areas, Hurley staging at the time of interview $(r=-0.351 ; p=0.079)$, the number of comorbidities, or any comorbidities except for depression $(r=0.450 ; p=0.021)$. Total BDI-21 and DLQI scores correlated $(r=0.509 ; p=0.008)$. Female gender $(p=0.004)$ was the only significant variable that significantly affected the total BDI-21 score using stepwise regression analysis.

\section{Diagnostic Delay}

Mean delay of HS diagnosis (i.e., start of symptoms to diagnosis of disease) was $13.7 \pm 10.0$ years. Diagnoses received prior to the HS diagnosis included abscesses (30.8\%; 8/26), folliculitis/furunculosis $(19.2 \% ; 5 / 26)$, and acne $(11.5 \% ; 3 / 26)$. However, no clear diagnosis existed for the majority of patients $(53.8 \% ; 14 / 26)$ before receiving an HS diagnosis.

\section{Impact on Workability}

Results from the WPAI-SHP questionnaire showed that, of those employed ( $n=16 ; 61.5 \%), 2(12.5 \%)$ missed a mean of $28.75 \mathrm{~h}$ from work during the past 7 days because of problems associated with HS. The rest of the employed patients $(n=14)$ reported no absence from work during the past 7 days. The results from the patient interview showed that, over the past year, the mean number of HS-related sick days was 14.2 days $(n=22)$. The number of sick days ranged from 0 to 120 days, with $50 \%$ of patients using no sick days. Of those reporting sick days, $81 \%$ estimated that their sick time ranged from 2 to 15 days. Two patients, both with Hurley stage III disease, reported 112 and 120 days of HS-related sick leave, respectively.

\section{Treatment}

At the time of the interview, $42.3 \%$ of the patients $(11 / 26)$ were managed at the university hospital, $30.8 \%$ $(8 / 26)$ at their own healthcare center, $3.8 \%(1 / 26)$ at a private practice, and $3.8 \%(1 / 26)$ at occupational healthcare. Five patients (19.2\%) were currently not seeking care for their condition. All patients had experienced local treatment (antiseptics, local antibiotics) or oral antibiotics. Thirteen patients $(50.0 \%)$ were either using or had used oral retinoids, either isotretinoin $(10 / 26 ; 38.5 \%)$ or acitretin $(4 / 26 ; 15.4 \%) ; 1$ patient had tried both. Only 1 patient had received biologic treatment in the past (adalimumab for the treatment of Crohn's disease). Oral pain medication, mainly nonsteroidal anti-inflammatory drugs (71\%), had been used by $26.9 \%$ of patients (7/26). Totally, $69.2 \%$ had undergone incision and drainage; $50.0 \%$ 

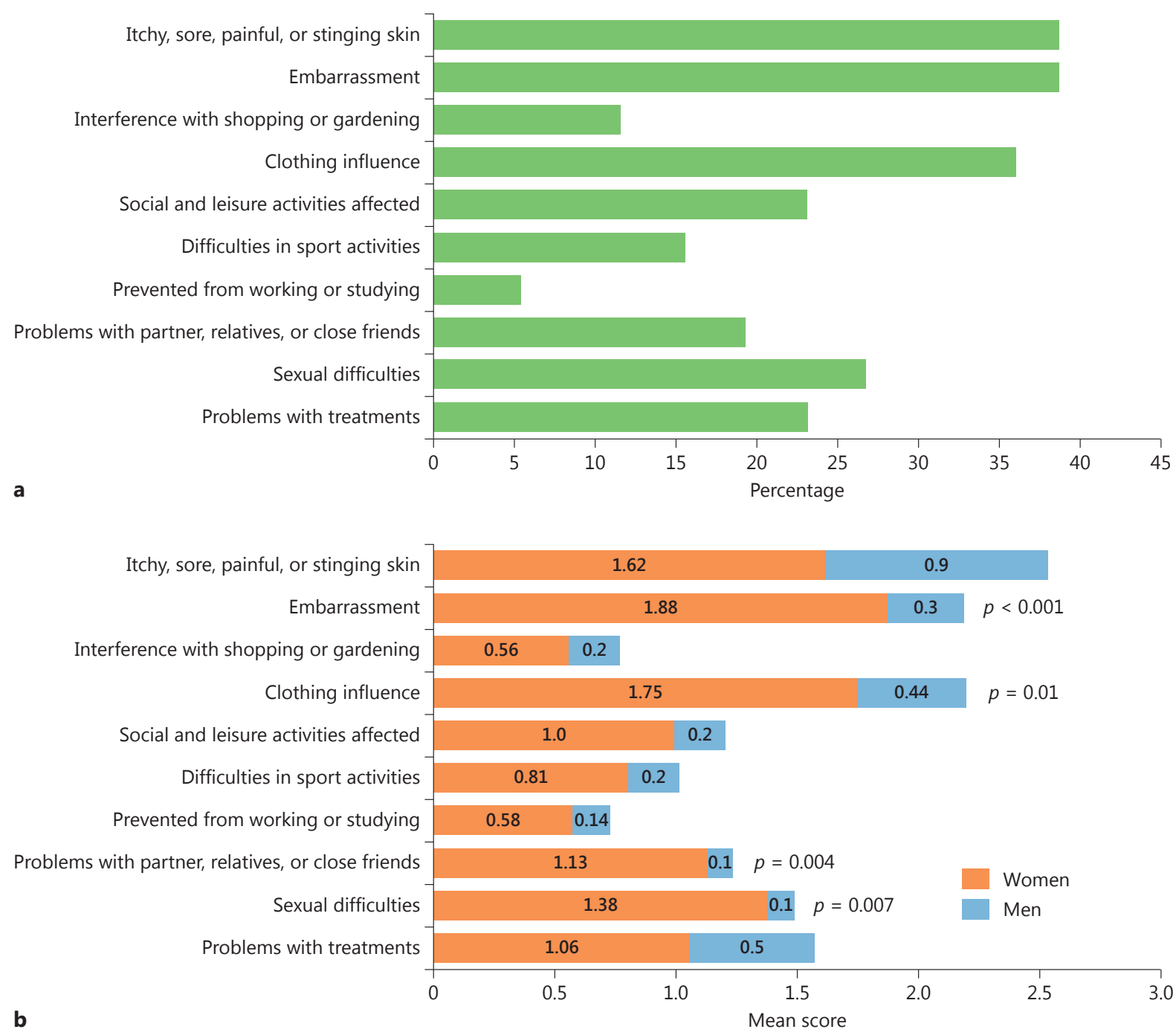

Fig. 3. a Impact of HS on each DLQI item. Data are presented as percentages of patients answering "a lot" or "very much" to each DLQI item. b Impact of HS on each DLQI item according to gender. Data are presented as mean scores for each DLQI question.

(13/26) already before their HS diagnosis. 23.1\% (6/26) still required these procedures occasionally. $19.2 \% \mathrm{had}$ undergone local excision and $11.5 \%$ wide excision.

\section{Discussion}

This is the first published report on the clinical characteristics and burden of disease in Finnish patients suffering from HS. Of note, a Scandinavian HS registry was recently established [8], but to our knowledge, it does not include any Finnish patients. We performed a cross-sectional pilot study on 26 patients in a single hospital district. Our findings are in line with current literature; a majority of patients were women and most were overweight or obese as well as smokers [9-11]. Family history was reported in approximately a third of the cases [12]. Surprisingly, we found an elevated mean delay of diagnosis of 13.7 years. This delay is longer than previously reported [11-13] and may have been caused by a delay in 
consulting a physician, the consulted physician not making the correct diagnosis, or both. The patient interviews indicated that the diagnostic delay was primarily caused by the healthcare provider. In the Finnish healthcare system, only the general practitioner in a local health center can decide on referral. This may play a significant role in diagnostic delay, especially in HS, where symptoms occur in cycles. The education of general practitioners in healthcare centers, from which the majority of referrals came, is crucial for timely referral to a specialist enabling accurate diagnosis.

Pain is reportedly an important aspect of HS. In the literature, HS-related pain has been described as "hot, burning, pressure, stretching, sharp, and splitting" [14]. In our study, pain was reported as the most disabling and bothersome symptom. The mechanism behind HS-related pain is still unknown and warrants further investigation $[15,16]$.

The impact of HS on QoL is well documented $[17,18]$. In our cohort, the mean DLQI score was 8.3, which is in line with results from other cohorts. Interestingly, in our study, women were clearly more affected than men in all aspects of the DLQI, especially embarrassment, clothing, sexuality, and social relationships. To our knowledge, this is the first report showing statistically significant differences in DLQI scores between men and women with HS. We report similar results regarding depression; according to BDI-21 scores, mainly women $(90.0 \%)$ reported symptoms of depression. Four patients (15.4\%) disclosed moderate to severe symptoms. The two patients with the highest BDI-21 total scores had received a diagnosis of depression before the diagnosis of HS. Due to the small sample size and the long diagnostic delay of HS reported in our cohort, it is difficult to draw a definitive conclusion on the relationship between depression and HS.

We assessed the impact of HS on workability in terms of sick days over the past year. Most patients tended not to take sick days because of HS. Only the most severely affected patients reported long sick leaves. It seems that Finnish patients, at least in our cohort, are quite resilient regarding their disease.

Our study has some limitations. It was a descriptive study without any control group. In addition, we cannot rule out the fact that the sample size $(n=26)$ may have affected our results. Nevertheless, our study showed similar results to other European and North American studies with regards to demographics, clinical presentation, comorbidities, and impact on QoL. Despite the limitations, our results are not based on a chart review only, but are supplemented with data from patient interviews, physical examinations, and patient questionnaires. The patient interview allowed the patient to provide his or her own valuable insight into the burden of HS on the QoL.

Even though our results largely confirm those of others, we report novel findings on diagnostic delay and inter-gender-specific differences in QoL. The surprisingly long mean delay in diagnosis highlights the need for education of general practitioners in Finnish healthcare centers, as they may enable early diagnosis by timely referral to dermatologists. The QoL of patients suffering from HS, as measured by DLQI, is clearly impaired. In this pilot study, both DLQI and BDI-21 scores suggest that there is a clear difference between men and women, with women being more impacted by the disease. In the near future, we aim at extending this study to a nationwide scale.

\section{Acknowledgements}

The authors wish to thank Juha Turunen, $\mathrm{PhD}$, of Farenta Oy for data management services and Teppo Huttunen of 4 Pharma for conducting the statistical analyses. The authors also wish to thank Jenna Olkkonen, registered nurse, for her assistance and the patients for taking part in this study. Martha Skup, PhD, of AbbVie is acknowledged for the valuable input during the review of the manuscript.

\section{Statement of Ethics}

The Ethical Committee of HUCH Internal Medicine approved the study (191/13/03/01/15) and each participant provided informed consent.

\section{Disclosure Statement}

Dr. Nicolas Kluger has served as a consultant to AbbVie, and has received research funding from AbbVie and speaker fees from AbbVie and Galderma. Martta Ranta and Martina Serlachius are employees of AbbVie. The design, study conduct, and financial support for the study were provided by AbbVie. AbbVie participated in the interpretation of data, review, and approval of the publication.
Kluger/Ranta/Serlachius 


\section{References}

1 Revuz J: Hidradenitis suppurativa. J Eur Acad Dermatol Venereol 2009;23:985-998.

2 Jemec GB, Kimball AB: Hidradenitis suppurativa: epidemiology and scope of the problem. J Am Acad Dermatol 2015;73(5 suppl 1):S4-S7.

3 Zouboulis CC, Desai N, Emtestam L, Hunger RE, Ioannides D, Juhász I, Lapins J, Matusiak L, Prens EP, Revuz J, Schneider-Burrus S, Szepietowski JC, van der Zee HH, Jemec GB: European S1 guideline for the treatment of hidradenitis suppurativa/acne inversa. J Eur Acad Dermatol Venereol 2015;29:619-644.

4 Finlay AY, Khan GK: Dermatology Life Quality Index (DLQI) - a simple practical measure for routine clinical use. Clin Exp Dermatol 1994;19:210-216.

5 Dermatology Quality of Life Index (DLQI). http://www.dermatology.org.uk/quality/ dlqi/quality-dlqi-languages.html (accessed November 26, 2016).

6 Beck AT, Ward CH, Mendelson M, Mock J, Erbaugh J: An inventory for measuring depression. Arch Gen Psychiatry 1961;4:561571.

7 Beck AT: Measuring depression: The depression inventory; in Williams TA, Katz MM, Shield JA (eds): Recent advances in the psychobiology of the depressive illnesses. Washington, DC: Government Printing Office, 1972, pp 299-302.
8 Ingvarsson G, Dufour DN, Killasli H, Sartorius K, Lapins J, Skau PA, Moseng D, Dinparvar D, Furberg AS, Jemec GB, Emtestam L: Development of a clinical Scandinavian registry for hidradenitis suppurativa; HISREG. Acta Derm Venereol 2013;93:350351.

9 Revuz JE, Canoui-Poitrine F, Wolkenstein P, Viallette C, Gabison G, Pouget F, Poli F, Faye O, Roujeau JC, Bonnelye G, Grob JJ, BastujiGarin S: Prevalence and factors associated with hidradenitis suppurativa: results from two case-control studies. J Am Acad Dermatol 2008;59:596-601.

10 Shlyankevich J, Chen AJ, Kim GE, Kimball AB: Hidradenitis suppurativa is a systemic disease with substantial comorbidity burden: a chart-verified case-control analysis. J Am Acad Dermatol 2014;71:1144-1150.

11 Kim WB, Sibbald RG, Hu H, Bashash M, Anooshirvani N, Coutts P, Alavi A: Clinical features and patient outcomes of hidradenitis suppurativa: a cross-sectional retrospective study. J Cutan Med Surg 2016;20:52-57.

12 Saunte DM, Boer J, Stratigos A, Szepietowski JC, Hamzavi I, Kim KH, Zarchi K, Antoniou C, Matusiak L, Lim HW, Williams M, Kwon HH, Gürer MA, Mammadova F, Kaminsky A, Prens E, van der Zee HH, Bettoli V, Zauli S, Hafner J, Lauchli S, French LE, Riad H, ElDomyati M, Abdel-Wahab H, Kirby B, Kelly
G, Calderon P, del Marmol V, Benhadou F, Revuz J, Zouboulis CC, Karagiannidis I, Sartorius K, Hagströmer L, McMeniman E, Ong N, Dolenc-Voljc M, Mokos ZB, Borradori L, Hunger RE, Sladden C, Scheinfeld N, Moftah N, Emtestam L, Lapins J, Doss N, Kurokawa I, Jemec GB: Diagnostic delay in hidradenitis suppurativa is a global problem. Br J Dermatol 2015;173:1546-1549.

13 Mebazaa A, Ben Hadid R, Cheikh Rouhou R Trojjet S, El Euch D, Mokni M, Zitouna M, Ben Osman A: Hidradenitis suppurativa: a disease with male predominance in Tunisia. Acta Dermatovenerol Alp Pannonica Adriat 2009;18:165-167.

14 Smith HS, Chao JD, Teitelbaum J: Painful hidradenitis suppurativa. Clin J Pain 2010;26: 435-444.

15 von der Werth JM, Jemec GB: Morbidity in patients with hidradenitis suppurativa. Br J Dermatol 2001;144:809-813.

16 Ring HC, Sørensen H, Miller IM, List EK, Saunte DM, Jemec GB: Pain in hidradenitis suppurativa: a pilot study. Acta Derm Venereol 2016;96:554-556.

17 Gooderham M, Papp K: The psychosocial impact of hidradenitis suppurativa. J Am Acad Dermatol 2015;73(5 suppl 1):S19-S22.

18 Deckers IE, Kimball AB: The handicap of hidradenitis suppurativa. Dermatol Clin 2016; 34:17-22.
Hidradenitis Suppurativa in a Finnish Cohort
Skin Appendage Disord 2017;3:20-27 